\title{
RESISTENCIA A LA ENFERMEDAD DE CRÍA YESIFICADA POR COLONIAS DE APIS MELLIFERA CON EFICIENTE COMPORTAMIENTO HIGIÉNICO (HYMENOPTERA, APIDAE)
}

\author{
Ciro Invernizzi ${ }^{1}$
}

\begin{abstract}
CHALKBROOD DISEASE RESISTANCE IN APIS MELLIFERA COLONIES WITH EFICIENT HYGIENIC BEHAVIOUR (HYMENOPTERA, APIDAE). In an apiary composed of 14 hygienic and 7 non-hygienic colonies of Apis mellifera Linnaeus, 1758 the presence of visible and capped mummies was recorded, one hygienic and 4 non-hygienic colonies showed symptoms of chalkbrood. Twenty-eight days after a massive contamination of the colonies with pollen patties containing Ascosphaera apis Olive \& Spiltoir, 1955, the situation was almost identical to that at the beginning: the same 4 non-hygienic colonies still were infected and one hygienic colony that was healthy became infected. The high proportion of hygienic colonies that eliminated the disease symptoms suggests that they could maintain themselves healthy in spite of the presence of colonies with chalkbrood in the apiary.
\end{abstract}

KEYWORDS. Honey bees, Apis, chalkbrood, Ascosphaera, hygienic behaviour.

\section{INTRODUCCIÓN}

En las abejas Apis mellifera Linnaeus, 1758 la enfermedad de Cría Yesificada o Ascosferiosis es causada por el hongo heterotálico Ascosphaera apis Olive \& Spiltoir, 1955. Las larvas ingieren las esporas junto con su alimento y estas germinan en el extremo distal del intestino, en el momento en que las larvas comienzan a ser operculadas (8 a 9 días de edad). Los micelios se expanden rápidamente, atraviesan la membrana peritrófica y tres días después llegan a la superficie de la larva continuando su crecimiento en forma aérea. En este momento las larvas aparecen como estructuras duras normalmente de color blanco, llamadas yesos o momias, que eventualmente puede tornarse gris o negro si se forman cuerpos fructíferos (BAMFORD \& HEATH, 1989; BAILEY \& BALL, 1991).

La Cría Yesificada se encuentra distribuida en casi todo el mundo y en Uruguay se ha incrementado mucho en los últimos años siendo frecuente encontrar colonias sanas y enfermas en los apiarios de producción, fundamentalmente durante la primavera y el verano. La dispersión de $A$. apis entre colonias de un apiario es muy fácil debido a la deriva de abejas, al robo de miel y adicionalmente al intercambio de materiales de la colmena por los apicultores (MeHr et al., 1976; HerberT et al., 1977; KoENIG et al., 1987).

Los esfuerzos por controlar esta enfermedad con antimicóticos y otros químicos presentan muchos problemas debido a su dificil y costosa aplicación en la colmena y, eventualmente a los efectos secundarios negativos sobre la cría, abejas adultas y miel (GILLIAM \& V ANDENBERG, 1990). El uso de abejas seleccionadas para manifestar un eficiente comportamiento higiénico aparece como una alternativa en el control de la enfermedad (Milne, 1983; Gilliam et al., 1983; Gilliam et al., 1988; SpivaK \& Gilliam, 1993; SpivaK \& REUTER, 1998). Este mecanismo de resistencia comportamental consta de dos etapas: el desoperculado de la celda y la remoción de la pupa muerta. Cada etapa está gobernada

1. Sección Etología, Facultad de Ciencias. Iguá 4225, CP 14000, Montevideo, Uruguay. (ciro@fcien.edu.uy) 
por un gen recesivo, de modo que únicamente las abejas doble recesivas pueden realizar un comportamiento higiénico completo (ROTHENBUHLER, 1964). Para MoRITZ (1988) el patrón de herencia es más complejo incluyendo interacciones genéticas como la aditividad, epistasis y dominancia, frecuentes en la expresión de la mayoría de los comportamientos. La utilización de abejas higiénicas mostró en el pasado excelentes resultados en el control de la Loque Americana causada por la bacteria Paenibacillus larvae White, 1907 (PARK, 1937; WoOdrow, 1941; RothenbUHLER, 1964) y variables en el control del ácaro parásito Varroa jacobsoni Oudemans, 1904 (BoeCKING et al., 1992; SPIVAK, 1996).

En Uruguay los apiarios de producción están formados por colonias de abejas altamente hibridizadas, consecuencia de los apareamientos libres de abejas $A$. mellifera iberica Goetze, 1964 y A. mellifera ligustica Spinola, 1806 principalmente, e híbridos de $A$. mellifera scutellata Lepeletier, 1836 durante muchos años (BURGETT et al., 1995).

Se pretende determinar si el comportamiento higiénico es un componente de resistencia importante a la Cría Yesificada que permite a las colonias higiénicas, sin selección previa en este sentido, mantenerse sanas en apiarios que contienen colonias enfermas.

\section{MATERIAL Y MÉTODOS}

El experimento se realizó durante los meses de noviembre y diciembre de 1996 y enero de 1997 en un apiario formado por 21 colonias de abejas altamente hibridizadas, encabezadas por reinas jóvenes libremente apareadas y sin selección previa. En este período las colonias ubicadas en colmenas tipo Langstroth llegaron a su máximo desarrollo. La población de abejas adultas cubría al menos los 10 cuadros de la cámara de cría y la cría se encontraba distribuida en más de 4 cuadros. El abundante flujo de néctar permitió a las colonias acumular promedialmente $20 \mathrm{~kg}$ de miel y néctar en las alzas melarias.

Durante los meses de noviembre y diciembre se realizaron en cada colonia 4 pruebas sucesivas para evaluar el comportamiento higiénico. Para ello se mató aproximadamente 100 larvas recién operculadas pinchándolas a través del opérculo con un alfiler entomológico y se dejó el panal 24 horas dentro de la colmena (NEWTON \& OsTASIEWSKI, 1986). El número de celdas limpiadas por las abejas en este tiempo se expresó como Tasa de Limpieza (TL) en porcentaje. Antes de continuar con el experimento, todas las colonias fueron examinadas exhaustivamente para verificar la presencia de Cría Yesificada.

El día 3 de enero se procedió a contaminar experimentalmente a cada una de las colonias alimentándolas con $200 \mathrm{~g}$ de torta de polen conteniendo Ascosphaera apis. Para hacer las tortas primero se pulverizaron $2 \mathrm{~kg}$ de polen comercial junto con los yesos (4 yesos blancos y 4 yesos negros por colonia) en una licuadora. Luego se agregó un kg de azúcar y agua destilada hasta obtener una consistencia semisólida. Las tortas se colocaron sobre los cabezales de la cámara de cría y dos días después las abejas las habían consumido.

Luego de la contaminación masiva se realizaron 4 inspecciones los días 9, 13, 20 y 31 de enero. La enfermedad se registró como presencia o ausencia de yesos visibles u operculados. La presencia de yesos operculados se determinó desoperculando manualmente 250 celdas de un panal con abundantes yesos visibles (en caso que los hubiese) o de un panal cualquiera con cría recién operculada. Para el análisis se dividieron arbitrariamente las colonias en higiénicas y no higiénicas según sus valores de TL fueran mayores o menores al $80 \%$ respectivamente.

\section{RESULTADOS}

De acuerdo a las respuestas a la prueba de comportamiento higiénico el apiario quedó conformado por 14 colonias higiénicas y 7 no higiénicas (tab. I). La mayoría de las colonias del primer grupo mostraron una alta repetibilidad en las 4 evaluaciones del comportamiento higiénico, mientras que las colonias no higiénicas manifestaron su capacidad de limpieza en forma muy variable. De las 16 colonias que estaban sanas 
Tabla I. Comportamiento higiénico de las 21 colonias de abejas del apiario y la presencia de Cría Yesificada antes de la contaminación del día 3 de enero y durante las cuatro inspecciones siguientes realizadas ese mes. Abreviaturas: C, contaminación; H, colonias higiénicas; I, inspección; L, tasa de limpieza; NH, colonias no higiénicas; $\mathrm{O}$, yesos operculados; V, yesos visibles; +, presencia de yesos; 0 , ausencia de yesos.

\begin{tabular}{|c|c|c|c|c|c|c|c|c|c|c|c|c|}
\hline \multirow[t]{2}{*}{$\begin{array}{c}\text { Colonia } \\
\mathrm{N}^{\circ}\end{array}$} & \multirow[t]{2}{*}{$\begin{array}{c}\text { TL } \\
\text { Media } \pm \mathrm{DE}\end{array}$} & \multirow[t]{2}{*}{$\begin{array}{l}\text { Clasifi- } \\
\text { cación }\end{array}$} & \multicolumn{2}{|c|}{$\begin{array}{c}\mathrm{C} \\
3 \text { enero }\end{array}$} & \multicolumn{2}{|c|}{$\begin{array}{c}\text { I } \\
9 \text { enero }\end{array}$} & \multicolumn{2}{|c|}{$\begin{array}{c}\text { I } \\
13 \text { enero }\end{array}$} & \multicolumn{2}{|c|}{$\begin{array}{c}\text { I } \\
20 \text { enero }\end{array}$} & \multicolumn{2}{|c|}{$\begin{array}{c}\text { I } \\
31 \text { enero }\end{array}$} \\
\hline & & & V & $\mathrm{O}$ & $\mathrm{V}$ & $\mathrm{O}$ & $\mathrm{V}$ & $\mathrm{O}$ & $\mathrm{V}$ & $\mathrm{O}$ & V & $\mathrm{O}$ \\
\hline 5 & $97,8 \pm 0,3$ & $\mathrm{H}$ & 0 & 0 & + & 0 & 0 & 0 & 0 & 0 & 0 & 0 \\
\hline 20 & $97,7 \pm 2,3$ & $\mathrm{H}$ & 0 & 0 & 0 & 0 & 0 & 0 & 0 & 0 & 0 & 0 \\
\hline 17 & $93,7 \pm 9,4$ & $\mathrm{H}$ & 0 & 0 & 0 & 0 & 0 & 0 & 0 & 0 & 0 & 0 \\
\hline 22 & $93,1 \pm 4,5$ & $\mathrm{H}$ & 0 & 0 & + & 0 & 0 & 0 & 0 & 0 & 0 & 0 \\
\hline 8 & $93,1 \pm 6,2$ & $\mathrm{H}$ & 0 & 0 & + & + & 0 & 0 & 0 & 0 & 0 & 0 \\
\hline 15 & $93,0 \pm 2,8$ & $\mathrm{H}$ & 0 & 0 & + & 0 & 0 & 0 & 0 & 0 & 0 & 0 \\
\hline 1 & $93,0 \pm 5,6$ & $\mathrm{H}$ & 0 & 0 & + & + & + & 0 & 0 & 0 & 0 & 0 \\
\hline 26 & $92,0 \pm 4,8$ & $\mathrm{H}$ & 0 & 0 & 0 & 0 & 0 & 0 & 0 & 0 & 0 & 0 \\
\hline 4 & $90,8 \pm 10,8$ & $\mathrm{H}$ & 0 & 0 & + & 0 & 0 & 0 & 0 & 0 & 0 & 0 \\
\hline 10 & $89,1 \pm 4,3$ & $\mathrm{H}$ & + & + & + & + & + & + & + & + & 0 & 0 \\
\hline 11 & $85,7 \pm 14,4$ & $\mathrm{H}$ & 0 & 0 & + & 0 & 0 & 0 & 0 & 0 & 0 & 0 \\
\hline 25 & $84,8 \pm 13,6$ & $\mathrm{H}$ & 0 & 0 & + & 0 & 0 & 0 & 0 & 0 & 0 & 0 \\
\hline 16 & $82,6 \pm 17,0$ & $\mathrm{H}$ & 0 & 0 & 0 & 0 & 0 & 0 & 0 & 0 & 0 & 0 \\
\hline 19 & $82,0 \pm 15,3$ & $\mathrm{H}$ & 0 & 0 & + & + & + & + & + & + & + & + \\
\hline 12 & $77,1 \pm 13,7$ & $\mathrm{NH}$ & 0 & 0 & 0 & 0 & 0 & 0 & 0 & 0 & 0 & 0 \\
\hline 24 & $76,6 \pm 12,4$ & $\mathrm{NH}$ & + & 0 & + & + & 0 & 0 & + & + & + & 0 \\
\hline 23 & $71,7 \pm 25,7$ & $\mathrm{NH}$ & 0 & 0 & 0 & 0 & 0 & 0 & 0 & 0 & 0 & 0 \\
\hline 2 & $60,9 \pm 21,7$ & $\mathrm{NH}$ & + & + & + & + & + & + & + & + & + & + \\
\hline 18 & $57,2 \pm 20,7$ & $\mathrm{NH}$ & 0 & 0 & + & 0 & 0 & 0 & 0 & 0 & 0 & 0 \\
\hline 14 & $54,4 \pm 13,9$ & $\mathrm{NH}$ & + & + & + & + & + & + & + & + & + & + \\
\hline 9 & $49,4 \pm 13,4$ & $\mathrm{NH}$ & + & + & + & + & + & + & + & + & + & + \\
\hline
\end{tabular}

antes del 3 de enero, solo 9 colonias higiénicas $\left(\mathrm{N}^{\circ} 5,22,8,15,1,4,11,25\right.$ y 19) y la colonia $\mathrm{N}^{\circ} 18$ no higiénica se enfermaron luego de consumir las tortas de polen. En cambio, 4 colonias higiénicas $\left(\mathrm{N}^{\circ} 20,17,26\right.$ y 16) y dos no higiénicas ( $\mathrm{N}^{\mathrm{o}} 12$ y 23$)$ no mostraron síntomas de Cría Yesificada durante el período de inspección.

Se manifestó una clara asociación entre la presencia de Cría Yesificada y el bajo comportamiento higiénico de las colonias. Este fenómeno se verifica en dos situaciones. Previo a la contaminación del apiario había 4 colonias no higiénicas $\left(\mathrm{N}^{\circ} 24,2,14\right.$ y 9) y una higiénica $\left(\mathrm{N}^{\mathrm{o}} 10\right)$ con Cría Yesificada. Finalmente, en la última inspección, el apiario se presentaba prácticamente igual que al comienzo del experimento, ya que las 4 colonias no higiénicas anteriores continuaban enfermas y también presentaba yesos la colonia $\mathrm{N}^{\circ} 19$ higiénica, que no estaba enferma antes de iniciar la contaminación del apiario. El resultado del test de Chi cuadrado fue igual para las dos situaciones descritas $\left(\chi^{2}=7,00 ; \mathrm{gl}=1 ; \mathrm{P}<\right.$ $0,01)$.

Del experimento también se desprenden las siguientes observaciones: en la primera inspección luego de la inoculación, encontramos 6 colonias muy higiénicas $\left(\mathrm{N}^{\mathrm{o}} 5,22,15\right.$, $4,11,25)$ y una poco higiénica $\left(\mathrm{N}^{\circ} 18\right)$ que presentaban yesos visibles pero no operculados (menos de 0,4\%). En la segunda inspección estas colonias no presentaron síntomas de la enfermedad. Idéntica situación presentó la colonia $\mathrm{N}^{\mathrm{o}} 1$ higiénica en la segunda y tercera inspección. Hubo un solo caso de reinfección durante el período de inspección (colonia $\mathrm{N}^{\mathrm{o}} 24$, no higiénica). No se encontró ninguna colonia que tuviese yesos ocultos pero que no mostrara al mismo tiempo yesos visibles. 


\section{DISCUSIÓN}

Los resultados indican con claridad que el comportamiento higiénico es un mecanismo de resistencia muy eficaz a la Cría Yesificada que permite que las colonias higiénicas se mantengan sanas dentro de un apiario con colonias enfermas. Esto se confirma con la rápida recuperación de la mayoría de las colonias higiénicas a solamente 10 días de infectar todas las colonias.

Es posible que la velocidad con que se eliminaron los síntomas esté vinculado a la incidencia de dos factores ambientales. Por un lado, el gran flujo de néctar presente durante el período de estudio pudo estimular a las abejas a limpiar las celdas. Al respecto, THOMPSON (1964) у Момот \& RotHENBUHLER (1971) encontraron que el comportamiento higiénico de una colonia aumenta cuando hay ingreso de néctar a la colmena, seguramente debido a la necesidad de celdas limpias para almacenarlo. Otro factor que pudo jugar un papel importante en la rápida recuperación del apiario es la falta de enfriamiento de la cría. Para Flores et al. (1996) es muy dificil que la cría se enferme si no sufre un enfriamiento en el momento en que es operculada su celda. Seguramente éste enfriamiento no ocurrió en la cría de las colonias experimentales ya que habían muchas abejas adultas y durante el mes de enero la temperatura ambiente es de aproximadamente $30^{\circ} \mathrm{C}$ durante el día y $20^{\circ} \mathrm{C}$ durante la noche.

Gilliam et al. (1983), Gilliam et al. (1988) y SpivaK \& Gilliam (1993), luego de contaminar masivamente con A. apis un apiario compuesto de colonias higiénicas y no higiénicas, encontraron que la mayoría de las colonias higiénicas presentan pocos yesos en los panales de cría y mayor capacidad de remoción de los yesos. MiLne (1983) evaluando separadamente el desoperculado y la remoción de las colonias en cajas de laboratorio, halló que sólo esta última etapa se relaciona significativamente con la resistencia a la Cría Yesificada.

La presencia de colonias con Cría Yesificada dentro de un apiario es suficiente para que las demás colonias tomen contacto con A. apis. MeHR et al. (1976) encontraron que unas pocas colonias enfermas pueden rápidamente infectar las demás colonias sanas a través de la deriva de abejas o por el robo de miel entre colonias. También HERBERT et al. (1977) consiguieron infectar colonias sanas con solo agregar reinas, obreras y panales con cría abierta u operculada tomados de colonias enfermas. Estos investigadores lograron a su vez recuperar el hongo a partir de obreras y reinas de colonias que habían eliminado todos los síntomas de la enfermedad. Koenig et al. (1987) detectó A. apis en la carga de polen de obreras pecoreadoras y en fuentes de agua dentro del apiario indicando que estas pueden ser vías de transmisión. Por tanto, el comportamiento higiénico constituye un mecanismo importante de las colonias para el control de la enfermedad, pese al ingreso constante del hongo por las vías mencionadas. La presencia del hongo dentro de la colmena posibilita las reinfecciones o el agravamiento de la enfermedad cuando las condiciones para su desarrollo son favorables. En esta investigación se verificó un solo caso de reinfección (colonia $\mathrm{N}^{\circ} 24$, no higiénica).

Existen dos posibles razones, no excluyentes, para explicar que 4 colonias higiénicas y dos no higiénicas no mostraran síntomas de la enfermedad en la primera inspección. Una razón sería que las abejas pudieron retirar las yesos en los 6 días siguientes a la contaminación, pues aún las dos colonias no higiénicas presentaron valores de TL promedio elevados dentro de su grupo $(77,1 \%$ y $71,7 \%)$. Otra, es que las 
larvas de estas colonias posean mayor resistencia fisiológica a la enfermedad, impidiendo el desarrollo de los hongos. Ninguna de ellas estaba enferma antes de la contaminación y permanecieron sanas durante las cuatro inspecciones siguientes aún ante la presencia de polen contaminado. En este sentido, SPIVAK \& GILLIAM (1993) encontraron algunas colonias que presentaban elevada resistencia comportamental y fisiológica a la Cría Yesificada.

Resulta interesante verificar que 8 colonias ( 7 higiénicas y una no higiénica), previamente a la eliminación de los síntomas de la enfermedad, hayan pasado por una etapa de mostrar yesos visibles pero no operculados. Esto significaría que las abejas pudieron reconocer la presencia de la cría muerta y desopercularla, pero no retiraron los yesos de las celdas. Seguramente la forma y textura que tienen los yesos juega un papel importante en la remoción. GILLIAM \& VANDENBERG (1990) describieron el primer estado de la larva enferma como blanco, esponjoso y totalmente expandido dentro de la celda, adquiriendo la forma de ésta. Posteriormente comienza a secarse y contraerse, tornándose dura, momento en el cual algunas forman cuerpos fructíferos y adquieren un color gris o negro. Posiblemente las obreras no puedan remover los yesos muy adheridos a la celda por imposibilidad física o mecánica, debiendo esperar hasta que se contraigan y desprendan para removerlas.

La Cría Yesificada al igual que otras enfermedades como la Loque Americana y la Varroasis matan a las larvas cuando sus celdas han sido operculadas. La forma en que las obreras reconocen la muerte de la cría a través del opérculo puede basarse en la pérdida de temperatura (GRAMACHO et al., 1998) o en los olores despedidos por la cría muerta (SPivaK \& Downey, 1998; Gramacho et al., 1999).

La prueba de comportamiento higiénico ha sido aplicada con éxito en programas de selección de abejas que buscan aumentar la tolerancia a las enfermedades de la cría (Spivak \& Reuter, 1998; Palacio et al., 2000). También es destacable que es una prueba sencilla, fácil de realizar por parte de los apicultores para mejorar sus colonias.

Agradecimientos. A Marila Lázaro y Bettina Tassino por la lectura crítica del manuscrito. A Mario Torres por su ayuda en las tareas de campo. Al revisor anónimo por sus valiosas sugerencias para mejorar el trabajo.

\section{REFERENCIAS BIBLIOGRÁFICAS}

Bailey, L. \& Ball, B. V. 1991. Honey Bee Pathology. 2. ed. London, Academic. 193p.

Bamford, S. \& Heath, L. A. F. 1989. The infection of Apis mellifera larvae by Ascosphaera apis. J. Apic. Res., Louisiana, 28:30-35.

Boecking, O.; Rath, W. \& Drescher, W. 1992. Apis mellifera removes Varroa jacobsoni and Tropilaeps clarae from sealed brood cells in the tropics. Am. Bee J., Hamilton, 132:732-735.

Burgett, M.; Shorney, S. et al. 1995. The present status of africanized honey bees in Uruguay. Am. Bee J., Hamilton, 135:328-330.

FloRes, J. M.; Ruiz, J. A. et al. 1996. Effect of temperature and humidity of sealed brood on chalkbrood development under controlled conditions. Apidologie, Paris, 27:185-192.

Gilliam, M.; Taber, S. \& Richardson, G. V. 1983. Hygienic behavior of honey bees in relation to chalkbrood disease. Apidologie, Paris, 14:29-39.

Gilliam, M.; TABER, S. et al. 1988. Factors affecting development of chalkbrood disease in colonies of honey bees, Apis mellifera, fed pollen contaminated with Ascosphaera apis. J. Invertebr. Pathol., San Diego, 52:314-325.

Gilliam, M. \& Vandenberg, J. D. 1990. Fungi. In: Morse, R. A. \& Nowogrodzki, R. eds. Honey Bee Pest, Predators, and Diseases. 2. ed., Ithaca, Cornell University. p. 65-90. 
Gramacho, K. P.; Gonçalves, L. S. et al. 1998. Study of the temperature of brood killed by the pin-killing method in worker bees of Apis mellifera carnica. Apiacta, Bucarest, 33:33-41.

Gramacho, K. P.; Gonçalves, L. S. et al. 1999. Influence of body fluid from pin-killed honey bee pupae on higienic behavior. Apidologie, Paris, 30:367-374.

Herbert, E. W.; Shimanuki, H. \& Knox, D. A. 1977. Transmission of chalk brood disease of honeybees by infected queens, and worker brood and adults. J. Apic. Res., Louisiana, 16:204-208.

Koenig, J. P.; Boush, G. M. \& ERICKson, E. H., Jr. 1987. Isolation of the chalkbrood pathogen, Ascosphaera apis, from honey bee (Apis mellifera) surfaces, pollen loads, and a water sourse. Am. Bee J., Hamilton, 126: 581-583.

Mehr, Z.; Menapace, D. M. et al. 1976. Studies on the initiation and spread of the chalkbrood whithin an apiary. Am. Bee J., Hamilton, 116:266-268.

Milne, C. P. 1983. Honey bee (Hymenoptera: Apidae) higienic behavior and resistance to Chalkbrood. Ann. ent. Soc. Am., Lanham, 76:384-387.

Момот, J. P. \& RothenbuhLeR, W. C. 1971. Behaviour genetics of nest cleaning in honeybees VI. Interactions of age and genotipes of bees, and nectar flow. J. Apic. Res., Louisiana, 10:11-21.

MoRITZ, R. F. A. 1988. A reevaluation of the two locus model for higienic behavior in honeybees (Apis mellifera L.). J. Hered., Washington DC, 79: 257-262.

Newton, D. C. \& Ostasiewski, N. J. 1986. A simplified bioassay for behavioral resistance to American foulbrood in honey bees (Apis mellifera L.). Am. Bee J., Hamilton, 126: 278.

Palacio, M. A.; Figini, E. E. et al. 2000. Changes in a population of Apis mellifera L. selected for higienic behaviour and its relation to brood disease tolerance. Apidologie, Paris, 31:469-471.

PARK, O. W. 1937. Testing for resistance to American foulbrood in honeybees. J. econ. Ent., Lanham, 30: 504-512.

RothENBuHLER, W.C. 1964. Behavior genetics of nest cleaning in honey bees: IV. Responses of F1 and backcross generations to disease-killed brood. Am. Zool., Mc Lean, 4: 111-123.

SPIVAK, M. 1996. Hygienic behavior and defense against Varroa jacobsoni. Apidologie, Paris, 27: 245-260.

SPIVAK, M. \& DownEY, D. L. 1998. Field assays for hygienic behavior in honey bees (Apidae: Hymenoptera). J. econ. Ent., Lanham, 91: 64-70.

SPIVAK, M. \& GiLliam, M. 1993. Facultative expression of hygienic behaviour of honey bees in relation to disease resistance. J. Apic. Res., Louisiana, 32: 147-157.

SPIVAK, M. \& ReUTER, G. S. 1998. Perfomance of hygienic honey bee colonies in a commercial apiary. Apidologie, Paris, 29:291-302.

Thомpson, V.C. 1964. Behaviour genetics of nest cleaning in honeybees: III. Effect of age of bees of a resistant line on their response to disease-killed brood. J. Apic. Res., Louisiana, 3: 25-30.

Woodrow, A. W. 1941. Behavior of honeybees toward brood infected with American foulbrood. Am. Bee J., Hamilton, 81: 363-366.

Recebido em 10.10.2000; aceito em 05.06.2001 